\title{
Evidence for a novel cytoplasmic processing event in ribosome maturation in the sea urchin Paracentrotus lividus
}

\author{
Daniele Bellavia • Rainer Barbieri
}

Received: 20 October 2009/Revised: 11 January 2010/Accepted: 28 January 2010/Published online: 27 February 2010

(C) Springer Basel AG 2010

\begin{abstract}
In this paper we demonstrate the existence of a cytoplasmic processing step, never before described, involving both the pre-ribosomal subunits in the sea urchin Paracentrotus lividus. Northern-blot hybridization, primer extension, S1 mapping experiments and in situ hybridizations allowed us to demonstrate that cytoplasmic processed particles are successively re-imported into the nucleus where maturation of their RNAs is completed prior to being exported to the cytoplasm. Our findings lead to the proposal of a new model of ribosome maturation and shuttling.
\end{abstract}

Keywords Ribosome maturation · Processing ·

Shuttling $\cdot$ Sea urchin $\cdot$ Pre-rRNAs

\section{Introduction}

Ribosome building in eukaryotes involves both processing of pre-ribosomal RNA (pre-rRNA) and sequential assembly of numerous different ribosomal proteins (RP) on a primary rRNA precursor molecule. Ribosomal maturation requires a large number of ancillary proteins and small RNAs. These ancillary molecules associate and dissociate from pre-ribosome and pre-ribosomal subunits during their movement from the nucleolus (the site of primary assembly) via the nucleus, to their final destination in the

D. Bellavia $\cdot$ R. Barbieri $(\bowtie)$

Dipartimento di Biologia Cellulare e dello Sviluppo,

Università degli Studi di Palermo, V.le delle Scienze,

Edificio 16, 90128 Palermo, Italy

e-mail: barner@unipa.it cytoplasm [1]. Different steps of pre-ribosome maturation have been elucidated in recent years (reviewed in [1-11]), but some intriguing aspects of these processes remain unclear. Among these, two important questions that remain are: (1) why the two ribosomal sub-units have different fates during maturation, and (2) why the different steps in the ribosomal particle maturation occur in different cellular compartments.

The opening events of ribosome biogenesis occur in the nucleolus [4, 8, 12] where a newly synthesized polycistronic rRNA transcript (indicated as $35 \mathrm{~S}$ in yeast, the organism in which this process has been extensively studied) is complexed with RP to form the 90S pre-ribosome [7, 13]. The 35S rRNA primary precursor is cotranscriptionally processed at $3^{\prime}$ end by an endonuclease (RntI) [10, 14] which removes a large part of the $3^{\prime}$-external transcribed spacer ( $3^{\prime}$-ETS). Afterwards, prerRNA processing proceeds following two different pathways. In one the rRNA primary precursor is simultaneously processed by endonucleolitic cleavages in the $\mathrm{A} 0$ and $\mathrm{A}_{1}$ sites in the $5^{\prime}$-external transcribed spacer $\left(5^{\prime}\right.$-ETS), producing the mature $18 \mathrm{~S}$ rRNA $5^{\prime}$-end. A further cleavage in $\mathrm{A}_{2}$, in the internal transcribed spacer 1 (ITS-1) gives rise to a $20 \mathrm{~S}$ and a $27 \mathrm{~S}$ pre-rRNAs belonging to $43 \mathrm{~S}$ and $66 \mathrm{~S}$ preribosomal subunits, respectively [7]. The other pathway involves a first endonucleolitic cleavage in $\mathrm{A}_{2}$, giving rise to two pre-rRNAs belonging to the $43 \mathrm{~S}$ and $66 \mathrm{~S}$ pre-ribosomal subunits. Successive endonucleolitic cleavages in $\mathrm{A} 0$ and $\mathrm{A}_{1}$ lead to the formation of the mature $18 \mathrm{~S} 5^{\prime}$-end $[15,16]$. A detailed description of these pathways is reported in Figure 4 of [17].

The resulting pre-subunits undergo different fates: the smaller is exported to the cytoplasm [18] where it is processed into the mature $40 \mathrm{~S}$ subunit containing the mature $18 \mathrm{~S}$ rRNA molecule, while the larger is retained in the 
nucleus where rRNA processing is completed before being exported to the cytoplasm [19] where only minor structural rearrangements occur $[20,21]$.

The same overall pathway is thought to be common to all the eukaryotes.

In the sea urchin, the synthesis of ribosome components (rRNAs and RPs) is regulated during early development $[22,23]$. An intense synthesis of these components during oogenesis [24] is followed by a silencing of both rRNA and RP genes in the mature egg and, after fertilization, up to the hatching blastula stage. At this stage, transcription of the genes involved in the synthesis of ribosomal components is restored, remaining active almost up to the pluteus larval stage [22].

An intriguing feature of rRNA processing in this organism is the presence of a $21 \mathrm{~S}$ pre-rRNA (precursor of $18 \mathrm{~S}$ mature rRNA), containing the entire ETS region, in the cytoplasm of mature eggs [23, 25] in which both a $28 \mathrm{~S}$ and a $29 \mathrm{~S}$ pre-rRNA (precursors of $26 \mathrm{~S}$ mature rRNA) are also found (pers. obs., unpublished data).

In this paper we demonstrate the presence of a prerRNA processing step in the cytoplasm of the sea urchin Paracentrotus lividus never before described, involving the pre-RNAs of both the pre-40S and the pre-60S ribosomal subunits. Cytoplasmic pre-rRNA cleavages occur in both $21 \mathrm{~S}$ and $29 \mathrm{~S}$ pre-rRNAs, giving rise to further rRNA precursors. Cytoplasm-processed pre-ribosomal particles are then re-imported into the nucleus, where maturation of both the subunits (and of the corresponding rRNAs) is completed, prior to being definitively exported to the cytoplasm.

Therefore these findings lead to the proposal of a new pathway of ribosome maturation and shuttling.

\section{Materials and methods}

Oocytes, eggs, and embryos of the sea urchin Paracentrotus lividus were collected as described elsewhere [23]. These samples were resuspended in TKM (50 mM TRIS$\mathrm{HCl} \mathrm{pH} \mathrm{7.5;} 0.4 \mathrm{M} \mathrm{KCl} ; 18 \mathrm{mM} \mathrm{Mg}$ Acetate) and gently homogenized on ice using a dounce.

Nuclei from these samples were isolated and separated from the cytoplasm by centrifugation at $15,000 \mathrm{rpm}$ for $45 \mathrm{~min}$ in a Beckman Ultracentrifuge, using an SW28 rotor, loading the homogenate on a layer of $1.8 \mathrm{M}$ sucrose solution. After centrifugation, nuclear fractions were recovered as a visible pellet while cytoplasmic compounds remained in the supernatant.

Nuclear pellets and cytoplasmic supernatants were checked by spot hybridization for the presence of NTS (not transcribed spacer) sequences, to demonstrate both the presence of nuclei in the pellet fractions and the absence of nuclear contaminants in cytoplasmic supernatants (data not shown in this paper).

RNAs were extracted from isolated nuclei using the guanidine isothiocyanate procedure [26] and from cytoplasmic compound using the Trizol (Invitrogen) procedure according to manufacturer's protocol.

To obtain a very selective electrophoretic separation, RNAs were fractionated in denaturing gels [27] using the Voltage Gradient Gel Electrophoresis (VGGE) system [28, 29], and Northern-blot hybridizations of equal amounts $(10 \mu \mathrm{g})$ of VGGE-fractionated RNAs were carried out as described elsewhere [30]. Radio-labeled probes used in Northern-blot hybridizations were prepared by PCR amplification [27] of specific rDNA regions, in the presence of labeled deoxy-ribonucleotides.

Primer extension reactions were carried out in the presence of appropriate primers (see Table 1) and labeled deoxy-nucleotides using "SuperScript II Rnase $\mathrm{H}^{-}$Reverse Transcriptase" (Invitrogen), according to manufacturer's protocol. Primer extension reaction products were fractionated on $12 \%$ polyacrylamide denaturing gels [27] as described elsewhere [31], and exposed on an X-ray film.

S1 mapping experiments were carried out as described elsewhere [23], using DNA-labeled probes described in detail in Table 1.

On the basis of the P. lividus rDNA sequence reported elsewhere (EMBL accession number AM981272), the positions of both the probes we used in Northern-blot and S1 mapping analyses and of the oligonucleotides used in primer extension experiments is shown on the top of each figure (see "Results") and reported in detail in Table 1.

In situ hybridizations were carried out in oocytes, eggs and 2/4 blastomeres stage using proper, biotinylated probes, as described elsewhere [32, 33]. Hybridized samples were stained with $300 \mathrm{nM}$ DAPI for $10 \mathrm{~min}$ at room temperature. Confocal microscopy was performed with a Zeiss LSM-510 microscope (Carl Zeiss, Oberkochen, Germany) equipped with the same objectives, to confirm co-localization by Z-stack analysis. The acquired images were improved using Adobe Photoshop software (Adobe Systems Incorporated, San Josè, USA).

\section{Results}

Based on our previous observations of the presence of both a partially processed $29 \mathrm{~S}$ and $28 \mathrm{~S}$ rRNAs (and presumably a pre-60S subunit) and of an ETS-containing 21S rRNA (and presumably a pre-40S subunit) $[22,23]$ in the cytoplasm of $P$. lividus, we carried out Northern-blot hybridizations, primer extension and S1 mapping experiments of RNAs extracted separately from the cytoplasm and from nuclei in 
Table 1 Probes and oligonucleotides used in the experiments reported below (see Results)

\begin{tabular}{llll}
\hline Primer or probe name & Sequence & Nucleotides position & Length \\
\hline Oli 18S & $5^{\prime}$-atgcatggcttaatctttgag & From 754 to 733 & $21 \mathrm{nt}$ \\
Oli 26S & $5^{\prime}$ - taaattcagcgggtggtctc & From 3499 to 3480 & $19 \mathrm{nt}$ \\
ETS probe & & From 1 to 690 & $690 \mathrm{bp}$ \\
ITS2A probe & From 3123 to 3454 & $331 \mathrm{bp}$ \\
ITS2B probe & From 3091 to 3454 & $363 \mathrm{bp}$ \\
A0-A' probe & From 200 to 585 & $385 \mathrm{bp}$ \\
S1-18S probe & From -100 to +754 & $854 \mathrm{bp}$ \\
S1-26S probe & From 2856 to 3499 & $643 \mathrm{bp}$ \\
\hline
\end{tabular}
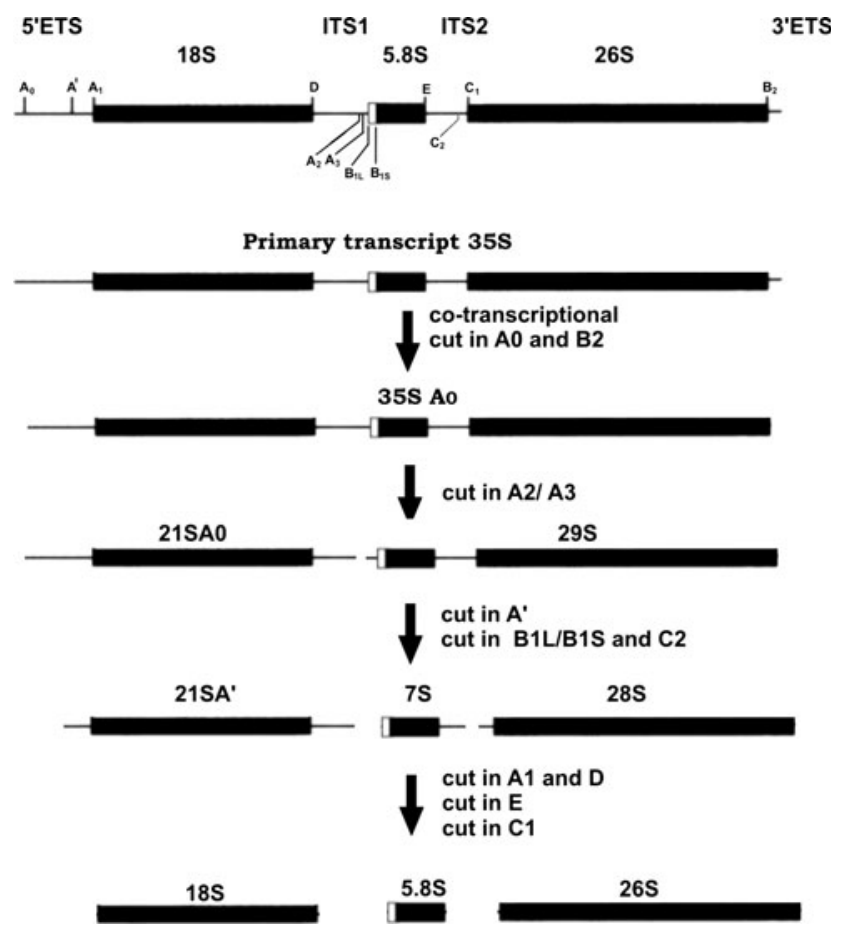

Fig. 1 Schematic drawing processing events of the large rRNAs in P. lividus

cells in which rRNA synthesis is active (oocytes and gastrula stage) and inactive (eggs and 2/4 blastomeres stage). Moreover, we carried out in situ hybridizations using confocal analysis to further demonstrate the localization of prerRNA during ribosome maturation.

On the basis of the data reported below, the processing events on 35S primary transcript in the sea urchin Paracentrotus lividus are summarized in Fig. 1.

Northern-blot hybridization, primer extension, and S1 mapping in "active" stages

Northern-blotted RNAs extracted from nuclei and cytoplasms of stages in which the synthesis of rRNA is active (oocytes and blastula stage) were hybridized with homologous labeled probes representing the ITS2 and the ETS
rDNA regions, respectively. Figure 1 shows the result of this experiment in which, apart from the presence of a nuclear $35 \mathrm{~S}$ primary precursor in RNAs extracted from the nuclei, identical hybridization patterns were observed both in the nuclei and the cytoplasm of these cells (oocytes and gastrula stage). In particular, partially processed $29 \mathrm{~S}, 28 \mathrm{~S}$, and $7 \mathrm{~S}$ pre-rRNAs both in the nucleus and in the cytoplasm (Fig. 2a, b), and an ETS-containing 21S pre-rRNA (Fig. 2e, f) were seen.

As the RNAs we used in this experiment were obtained from cells in which ribosomal RNA synthesis is active, the presence of $35 \mathrm{~S}$ pre-rRNA in the nuclear compounds was expected and represented an internal control showing that there had been no nuclear contamination of the RNAs extracted from the cytoplasm.

Northern data are supported by both primer extension and S1 mapping experiments (Fig. 2c, d, g, h), carried out on the same templates.

In primer extension analyses, we used an oligonucleotide primer complementary to an rRNA sequence 43 nucleotides downstream to the $5^{\prime}$-end of the mature $26 \mathrm{~S}$ rRNA (Fig. 2c), and an oligonucleotide primer complementary to a rRNA sequence 64 nucleotides downstream of the $5^{\prime}$-end of the mature 18S rRNA (Fig. 2g). In S1 mapping experiments we used radiolabeled probes (described in Table 1 of "Materials and methods") containing the $5^{\prime}$ end of 29S/28S/26S rRNAs (Fig. 2d) and of the 21SA0/ 21SA'/18S rRNAs (Fig. 2h), respectively.

Primer extension and S1 mapping data confirm the presence of $5^{\prime}$-ends of the same pre-rRNAs we identified in Northern-blot experiments, besides the presence of 5 -ends of the 26S (Fig. 2c) and the 18S (Fig. 2g) mature rRNAs both in the nuclei and cytoplasm of these cells.

Northern-blot hybridization, primer extension, and S1 mapping in "inactive" stages

Having previously demonstrated the lack of the synthesis of ribosomal components in P. lividus egg and, after fertilization, in early developmental stages [22, 23], we carried out the same analysis we had used on "active" 

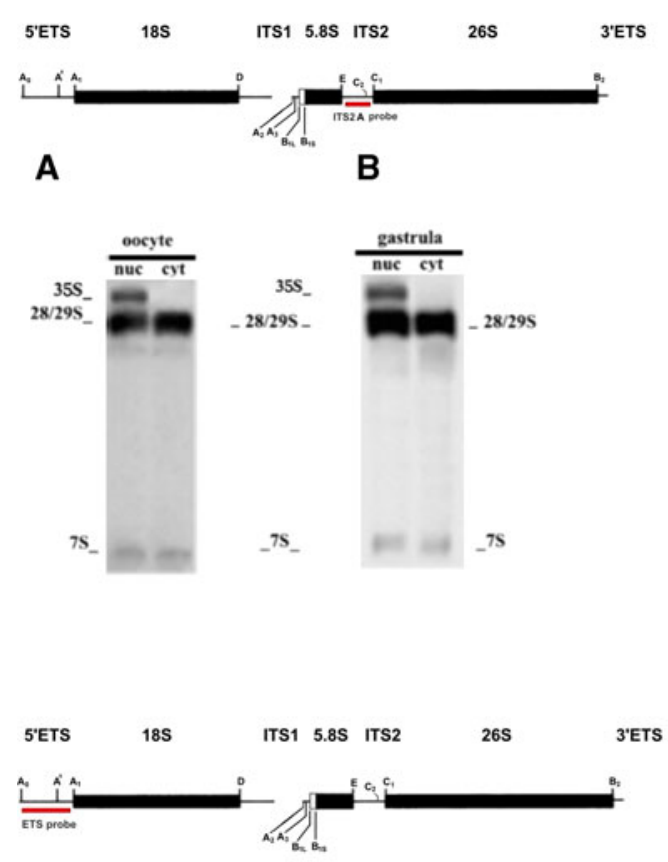

E

$\mathbf{F}$

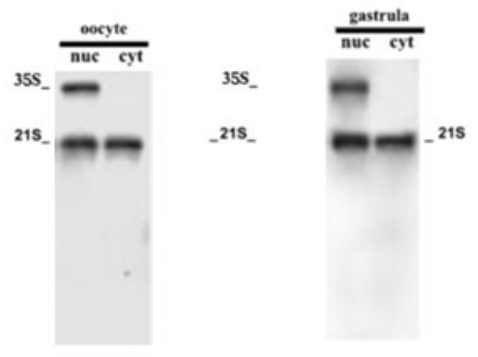

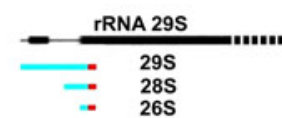

C
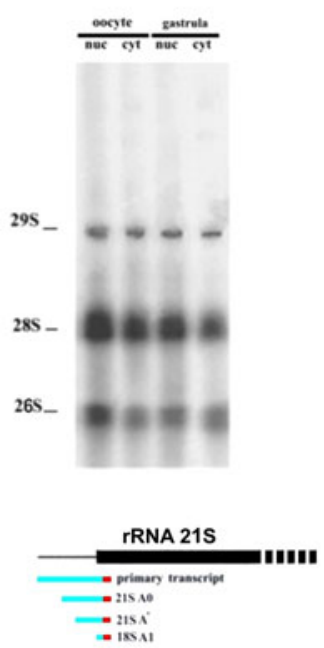

G

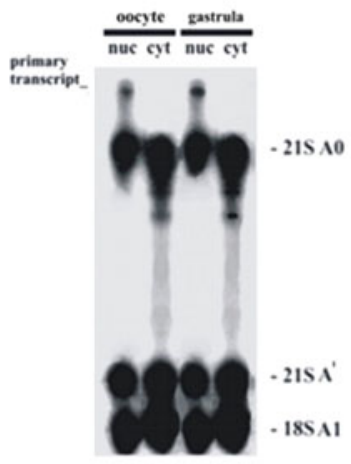

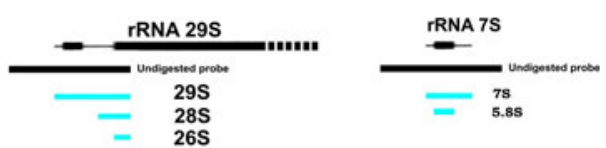

D

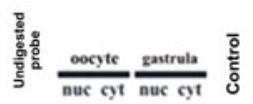
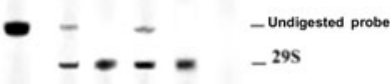

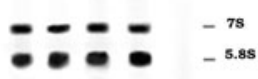

$\longrightarrow-285$

$-265$

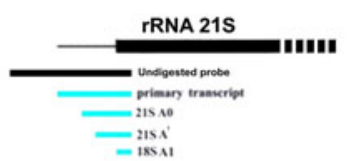

H
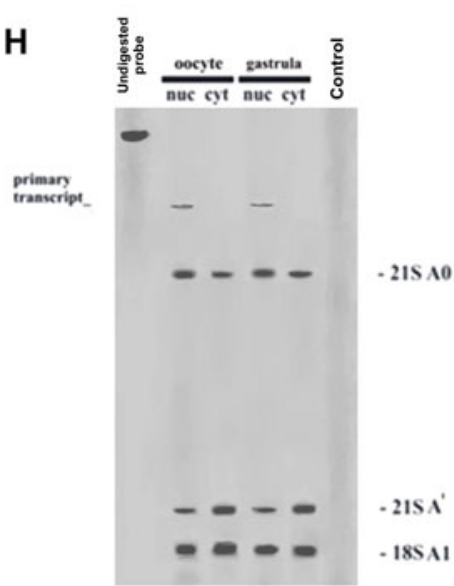

Fig. 2 Northern-blot hybridizations (a, b, e, f), primer extensions (c, $\mathbf{g}$ ), and S1 mapping (d, h) of RNAs extracted from nuclei (nuc) and cytoplasms (cyt) of different $P$. lividus cells (oocyte and gastrula stages), in which rRNA synthesis is active. Upper images (a-d) show RNAs belonging to the large subunit, whereas lower images (e-h) show RNAs belonging to the small subunit. A map of the probes used

stages, using the same probes and primers on these cells (Fig. 3).

Northern-blot hybridization experiments carried out on eggs and on $2 / 4$ blastomere stage (Fig. 3a, b, f, g) confirm the presence in the cytoplasm of $P$. lividus egg of all the rRNA secondary precursors we had found in "active" cells, while demonstrating that both precursors and mature rRNAs are absent from the nucleus of the egg. When we analyze Northern-blot hybridization patterns in the $2 / 4$ blastomeres stage (Fig. 3a, b, f, g), the existence of a processing step involving the ribonucleic component of both the ribosomal pre-subunits and the consequent in Northern-blot analyses $(\mathbf{a}, \mathbf{b}, \mathbf{e}, \mathbf{f})$ and the DNA fragments obtained in primer extension $(\mathbf{c}, \mathbf{g})$ and $\mathrm{S} 1$ mapping $(\mathbf{d}, \mathbf{h})$ experiments is shown above each image. The relatively weak signals observed in the primer extended $26 \mathrm{~S}$ and $18 \mathrm{~S}$ bands $(\mathbf{c}, \mathbf{g})$ are a consequence of the small number of labeled nucleotides incorporated in the very short "extended" regions belonging to these fragments

re-import of these processed particles into the nucleus is observed.

In the Northern-blot hybridization shown in Fig. 3b, a labeled probe (probe ITS2B) containing the entire ITS2 region and a few nucleotides of the $5.8 \mathrm{~S}$ coding region was used to map the presence of the 5.8S rRNA.

In this experiment, the presence of both the $29 \mathrm{~S}$ and the $28 \mathrm{~S}$ rRNA precursors (other than the $7 \mathrm{~S}$ and $5.8 \mathrm{~S}$ rRNAs) in the cytoplasm (Fig. 3b), corresponds to the presence of only a $28 \mathrm{~S}$ pre-rRNA (other than the $7 \mathrm{~S}$ and 5.8S rRNAs) in the nuclear compound (Fig. 3b), indicating that a cytoplasmic processing step occurs in the $29 \mathrm{~S}$ 


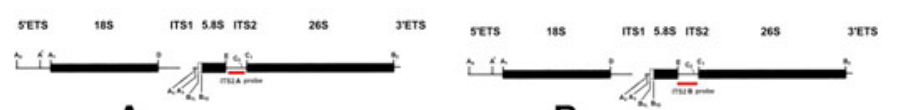

A

B
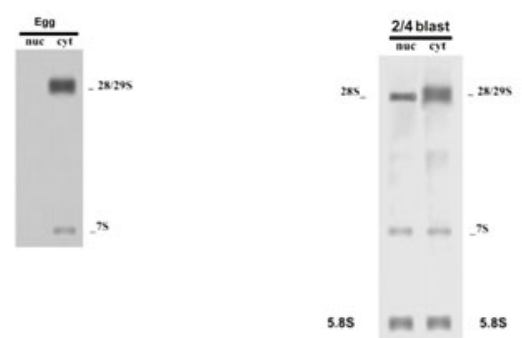

$\mathbf{F}$
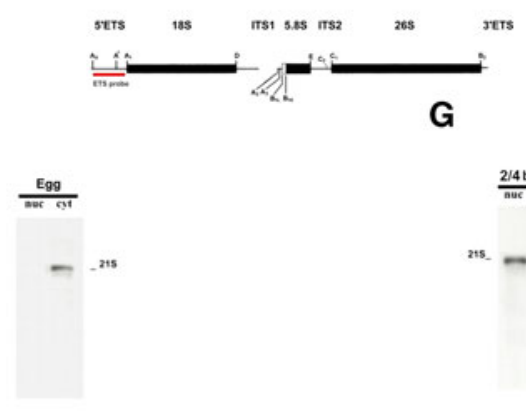

G

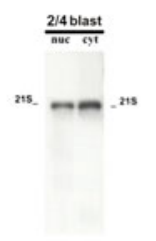

Fig. 3 The same experiments shown in Fig. 2 were carried out in "inactive" stages. Again, a map of the probes used in Northern-blot analyses $(\mathbf{a}, \mathbf{b}, \mathbf{f}, \mathbf{g})$ and the DNA fragments obtained in primer extension $(\mathbf{c}, \mathbf{d}, \mathbf{h}, \mathbf{i})$ and $\mathrm{S} 1$ mapping $(\mathbf{e}, \mathbf{j})$ experiments are shown. In the Northern-blot hybridization shown in $\mathbf{b}$, a labeled probe containing a few nucleotides of 5.8S rRNA coding sequence (probe ITS2B)

pre-rRNA, which produces a $28 \mathrm{~S}$ and a $7 \mathrm{~S}$ further rRNA precursors, and that only these last ones are re-imported into the nucleus.

Northern-blot analysis performed using the ETS-labeled probe (Fig. $3 \mathrm{~g}$ ) indicates the presence of a $21 \mathrm{~S}$ rRNA precursor both in the nucleus and in the cytoplasm at this stage.

Primer extension (Fig. 3c, d, h, i) and S1 mapping experiments (Fig. 3e, j) carried out on the same templates used in Northern-blot analyses, both confirm Northern data and indicate that a cytoplasmic processing event also occurs in the $21 \mathrm{~S}$ pre-rRNA, and that only a shortened, $\mathrm{A}^{\prime}$-processed $21 \mathrm{~S}$ pre-rRNA $\left(21 \mathrm{SA}^{\prime}\right)$ is imported into the nucleus (Fig. 3h, i, j).

In situ hybridizations in both "active" and "inactive" stages

In situ hybridizations (Fig. 4) carried out using the same (biotinylated) probes used in Northern-blot analyses demonstrate the presence of the same pre-ribosomal particles

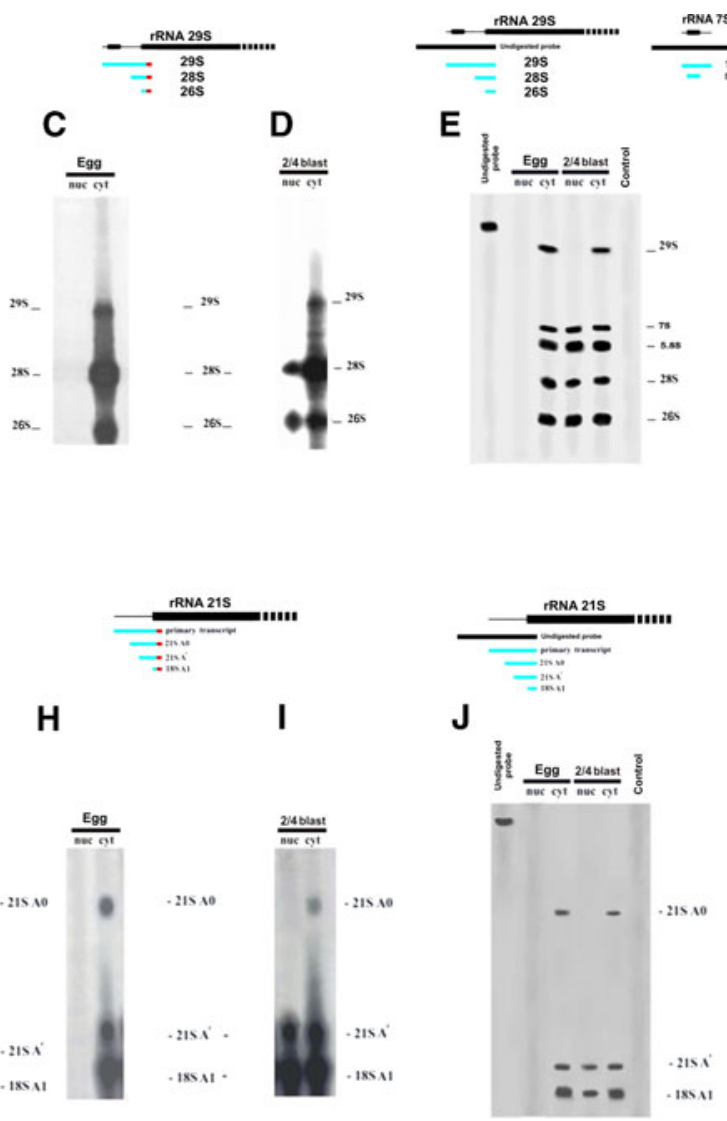

was used also, thus mapping the presence of $5.8 \mathrm{~S}$ mature rRNAs in both the compartments. The relatively weak signals observed in the primer extended $26 \mathrm{~S}$ and $18 \mathrm{~S}$ bands $(\mathbf{c}, \mathbf{d}, \mathbf{h}, \mathbf{i})$ are a consequence of the small number of labeled nucleotides incorporated in the very short "extended" regions belonging to these fragments

both in the nucleus and in the cytoplasm in the same stages analyzed in the experiments reported above (Figs. 2, 3).

Figure 4 shows the confocal microscopy result of these experiments carried out using the ETS probe (left) and the ITS2A probe (right).

In an "active stage" (oocytes), the presence of preribosomal particles both in the nucleus and in the cytoplasm is observed using both the probes (Fig. 4, first row). In unfertilized, mature eggs (Fig. 4, second row), the presence of the same pre-ribosomal particles are limited to the cytoplasm, and no hybridization signal is visible in the nucleus of these cells.

As occurred in "active" stages, in situ hybridizations carried out on egg and on the 2/4 blastomeres stage, using the ETS and the ITS2A probes, confirm the presence of pre-ribosomal particles in both the cellular compartments.

Moreover, to further demonstrate that the cytoplasmic processing event on the $21 \mathrm{~S}(\mathrm{~A} 0)$ pre-rRNA removes the $\mathrm{A} 0-\mathrm{A}^{\prime}$ fragment, as indicated by the primer extension and S1 mapping experiments shown in (Fig. $3 \mathrm{~h}, \mathrm{i}, \mathrm{j}$ ), and that 


\section{5'ETS \\ $18 S$ \\ ITS1 $5.8 \mathrm{~S}$ ITS2 \\ 26S \\ 3'ETS}
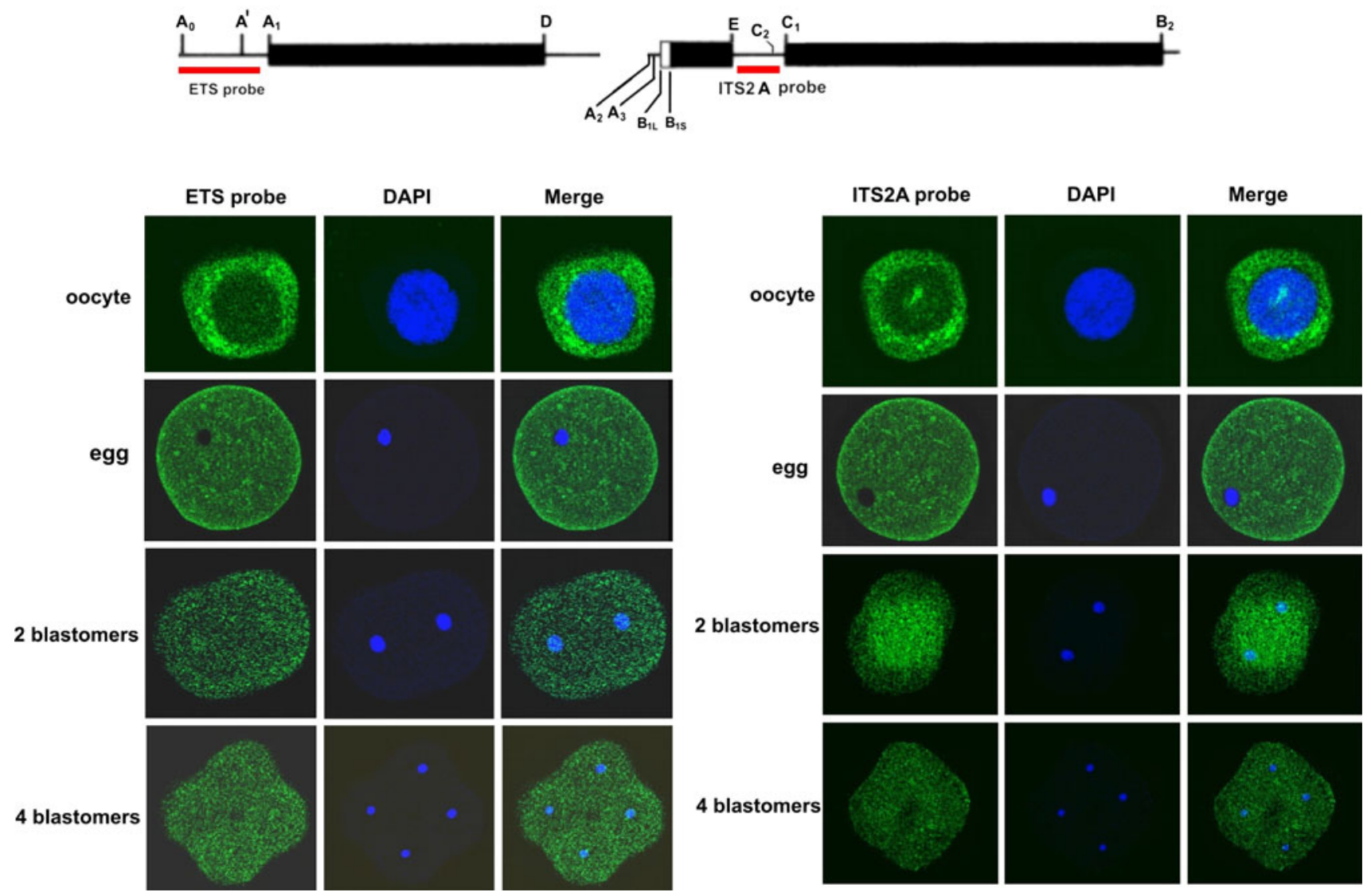

Fig. 4 Confocal microscopy of in situ hybridizations of the same stages analyzed in Figs. 3 and 4, using the ETS (left) and ITS2A (right) biotinylated probes, respectively

only this $\mathrm{A}^{\prime}$-processed pre-rRNA is re-imported into the nucleus, we carried out both Northern-blot and in situ hybridizations, using the $\mathrm{A} 0-\mathrm{A}^{\prime}$ fragment as a probe. Figure 5 shows the result of this analysis, and confirms the previous primer extension and S1 mapping data.

With respect to the $3^{\prime}$-end of this shortened $21 \mathrm{~S}$ prerRNA, RT-PCR experiments (not shown) carried out on total RNA extracted both from the nucleus and the cytoplasm of 2/4 blastomeres stage, using a reverse primer complementary to the $21 \mathrm{~S}\left(\mathrm{~A}_{2}\right) 3^{\prime}$-end and a direct primer corresponding to the $3^{\prime}$ of $18 \mathrm{~S}$ rRNA coding region, produces an amplified fragment of the same length from RNA of both the compartments, indicating that no cytoplasmic processing event occurs that involves the $3^{\prime}$ end of this pre-rRNA.

\section{Discussion}

The common sea urchin resulted an appropriate system for the study of pre-ribosomal subunit nucleus-cytoplasm maturation and shuttling. In sea urchin, both an abundant synthesis of ribosomes during oogenesis [24] and an almost constant ribosomal RNA content during early development [34, 35] were previously demonstrated.

The main peculiarity of the sea urchin with respect to ribosome maturation is the storage of the precursor ribosomal particles in the cytoplasm of mature eggs, when the primary rRNA precursor (35S) synthesis is silenced.

In unfertilized $P$. lividus eggs, the storage of both mature and precursor ribosomal particles occurs only in cytoplasmic compartment, allowing us to observe the processing steps and the movement of processed molecules after fertilization.

According to our findings a parallel, concerted processing/shuttling events involve both the pre-ribosomal subunits (and their pre-rRNAs), and leads to the pathway described in Fig. 6.

The first step of this pathway, suggested by the experiments shown in Figs. 2 and 3, is the export from the nucleus to the cytoplasm of both the pre-40S and pre-60S ribosomal particles. These particles contain a $21 \mathrm{~S}$ rRNA (A0-ETS region, 18S rRNA sequence and ITS1- $\mathrm{A}_{2}$ ) and a 
Fig. 5 Northern-blot hybridization (left) of RNA extracted from nuclei and cytoplasms of the $2 / 4$ blastomere ("inactive") stage using the $\mathrm{A} 0-\mathrm{A}^{\prime}$-labeled fragment as a probe. The same (biotinylated) probe was used in in situ hybridization confocal analyses (right) in the "active" (oocytes) and the "inactive" egg and two blastomere $P$. lividus stages
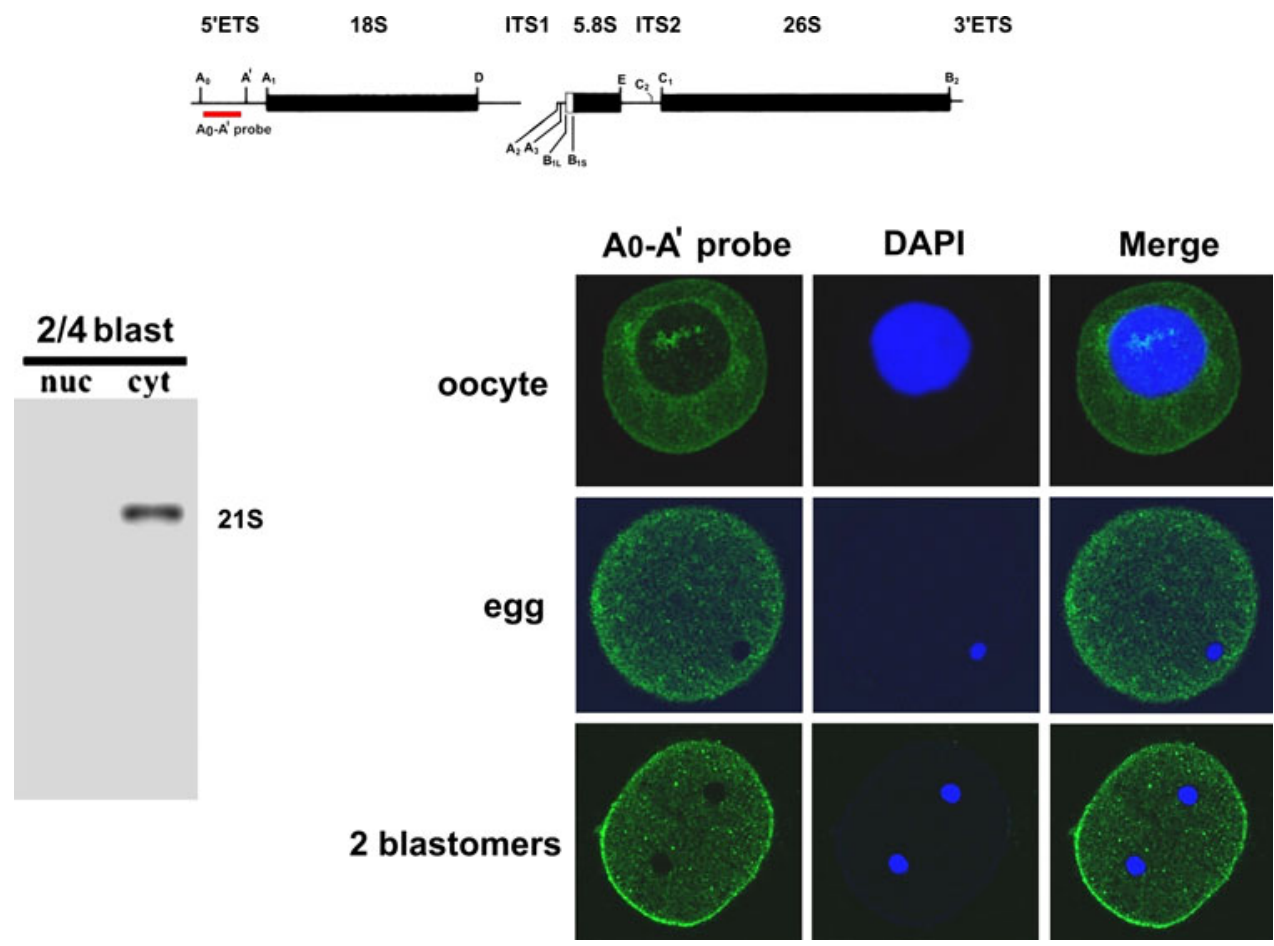

\section{Nucleus}
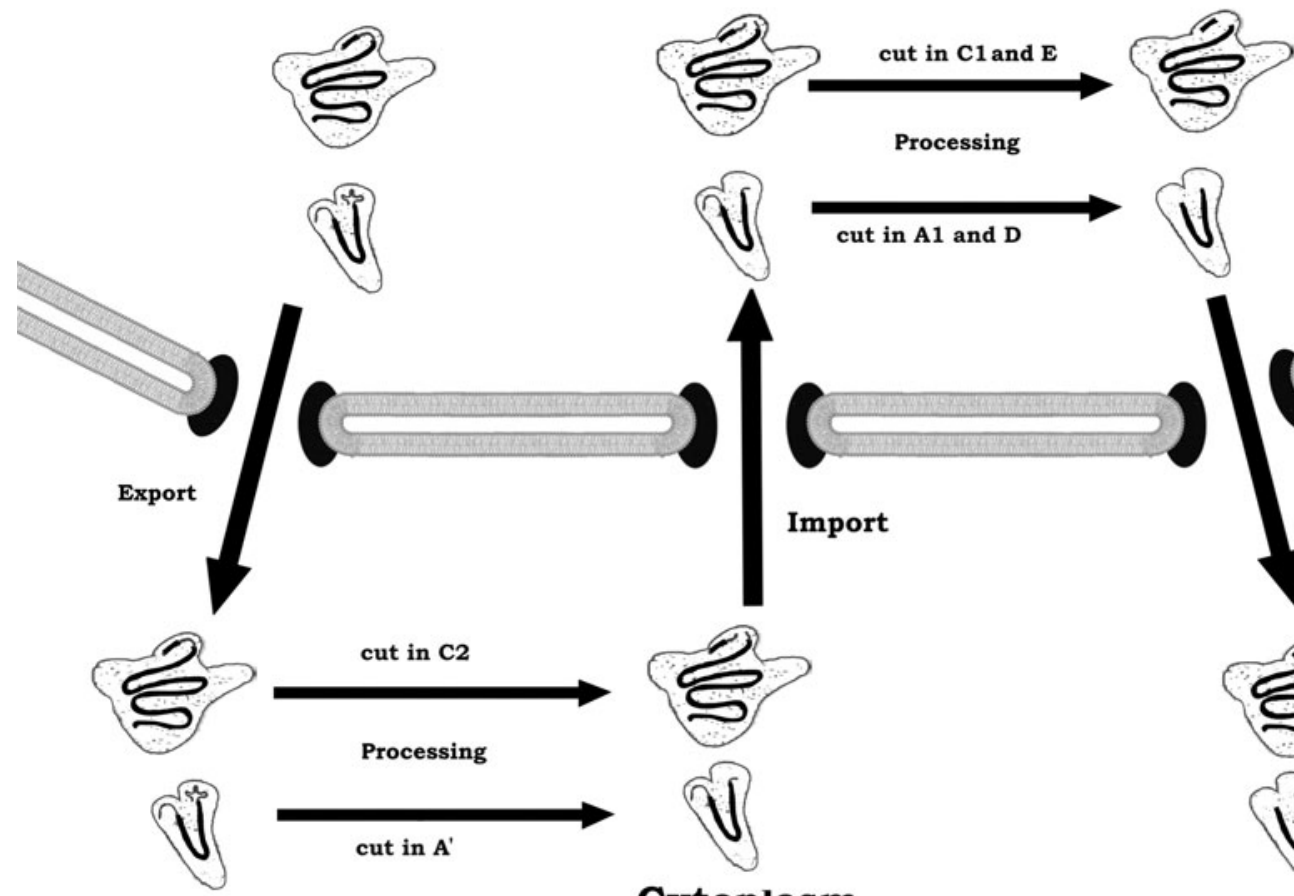

Import
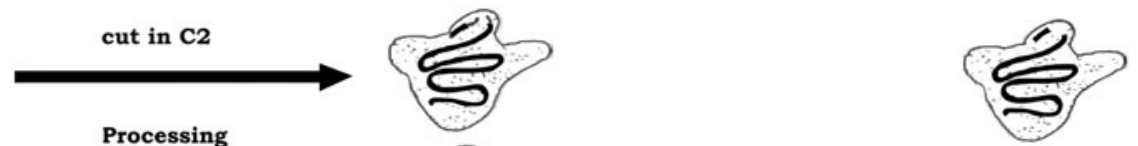

cut in $\mathbf{A}^{\prime}$
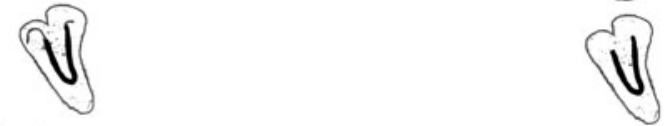

\section{Cytoplasm}

Fig. 6 The proposed ribosome maturation pathway based on the findings reported in this paper

29S rRNA ( A $_{2}$-ITS1 region, 5.8S rRNA sequence, ITS2, 26S rRNA sequence) respectively (personal, unpublished pre-rRNA mapping data). The RNA compounds of both these pre-subunits undergo a processing event which can be summarized as follows: in the pre-40S subunit, the $21 \mathrm{~S}$ rRNA (21SA0) is processed in $\mathrm{A}^{\prime}$ (a processing site never before described, unless it corresponds to the one described by Rouquette et al. [36] in Hela cells) producing a 
shortened RNA (that we indicated as $21 \mathrm{SA}^{\prime}$ ) lacking of the A0- $\mathrm{A}^{\prime}$ segment, indistinguishable from the former in Northern-blot analysis but distinguishable by primer extension and $\mathrm{S} 1$ mapping (see Fig. 3g, i, j). In the meantime, the 29S rRNA belonging to the pre-60S subunit is also processed in ITS2 (the $\mathrm{C}_{2}$ site in Fig. 1), giving rise to $28 \mathrm{~S}$ and $7 \mathrm{~S}$ pre-rRNAs (see Fig. $3 \mathrm{~b}$, d, e). Following fertilization, only these processed pre-subunits are then re-imported into the nucleus, where their maturation (and the processing of the correspondent rRNAs) is completed at $5^{\prime}$-ends, as demonstrated by the presence of mature $26 \mathrm{~S}$, $5.8 \mathrm{~S}$ and $18 \mathrm{~S}$ rRNAs in the nucleus of sea urchin $2 / 4$ cell embryos (Fig. 3). Mature particles are then exported to the cytoplasm, probably in the way previously described [37-41].

In the unfertilized egg, the presence of both unprocessed and processed RNAs belonging to pre-40S and pre-60S particles is demonstrated, and an arrest of the nuclear importation of the processed particles, active during oogenesis (see Fig. 2), is operative, since no ribonucleic compound was found in the nuclear compartment (see Fig. 3). At the two blastomeres stage, these processed particles are shown to re-enter the nucleus (Fig. 3), indicating that there is a releasing mechanism that allows the importation of the cytoplasm-processed pre-ribosomal particles. This mechanism seems to be directly related to the first zygotic division (personal data, not shown in this paper).

The presence of the ribosomal RNA precursors identified by Northern hybridizations, primer extensions and S1 mapping, is also confirmed by confocal microscopy observations (Figs. 4, 5).

Although we have neither isolated nor characterized any processing factor (and/or machinery), both the presence of processing events in the cytoplasm of $P$. lividus on the two pre-rRNA and the importation of cytoplasm-processed particles into the nucleus is unquestionable, as well as the subsequent nuclear maturation of the re-imported prerRNAs.

Preliminary data from our laboratory (not shown) suggest that the maturation pathway we propose in this paper (Fig. 6) may not be unique to the sea urchin, but a common feature in eukaryotes.

In the light of our findings, both ribosome biogenesis and rRNA processing events should be reinvestigated, and the pre-subunits maturation steps series should be revised according to the concerted processing/shuttling of preribosomal particles we propose here.

Acknowledgments We are deeply indebted to Professor David Tollervey for his kindness and illuminating suggestions. We are also indebted to Professor Nicholas Jerome Hyde for revising the English form. This work was supported by funds of the Italian M.I.U.R. (ex $60 \%)$.
Conflict of interest statement The authors declare that they have no conflict of interest.

\section{References}

1. Robledo S, Idol RA, Crimmins DL, Ladenson JH, Mason PJ, Bessler M (2008) The role of human ribosomal proteins in the maturation of rRNA and ribosome production. RNA 14:1918-1929

2. Fatica A, Tollervey D (2002) Making ribosomes. Curr Opin Cell Biol 14:313-318

3. Fromond-Racine M, Senger B, Saveanu C, Fasiolo F (2003) Ribosome assembly in eukaryotes. Gene 313:17-42

4. Gerbi SA, Borovjagin AV, Lange TS (2003) The nucleolus: a site of ribonucleoprotein maturation. Curr Opin Cell Biol 15:318-325

5. Granneman S, Baserga SJ (2004) Ribosome biogenesis: of knobs and RNA processing. Exp Cell Res 269:43-50

6. Granneman S, Baserga SJ (2005) Crosstalk in gene expression: coupling and co-regulation of rDNA transcription, pre-ribosome assembly and pre-rRNA processing. Curr Opin Cell Biol 17:281-286

7. Kumar A, Warner JR (1972) Characterization of ribosomal precursor particles from HeLa cell nucleoli. J Mol Biol 63:233-246

8. Raska I, Koberna K, Malínský J, Fidlerová H, Masata M (2004) The nucleolus and transcription of ribosomal genes. Biol Cell 96:579-594

9. Tschochner H, Hurt E (2003) Pre-ribosomes on the road from the nucleolus to the cytoplasm. Trends Cell Biol 13:255-263

10. Venema J, Tollervey D (1999) Ribosome synthesis in Saccharomyces cerevisiae. Annu Rev Genet 33:261-311

11. Zemp I, Kutay U (2007) Nuclear export and cytoplasmic maturation of ribosomal subunits. FEBS Lett 581:2783-2793

12. Dez C, Houseley J, Tollervey D (2006) Surveillance of nuclearrestricted pre-ribosomes within a subnucleolar region of Saccharomyces cerevisiae. EMBO J 25:1534-1546

13. Grandi P, Rybin V, Bassler J, Petfalski E, Strauss D, Marzioch M, Schäfer T, Kuster B, Tschochner H, Tollervey D, Gavin AC, Hurt E (2002) $90 \mathrm{~S}$ pre-ribosomes include the 35S pre-rRNA, the U3 snoRNP, and 40S subunit processing factors but predominantly lack 60S synthesis factors. Mol Cell 10:105-115

14. Allmang C, Tollervey D (1998) The role of the $3^{\prime}$ external transcribed spacer in yeast pre-rRNA processing. J Mol Biol 278:67-78

15. Borovjagin AV, Gerbi SA (2005) An evolutionary intra-molecular shift in the preferred U3 snoRNA binding site on preribosomal RNA. Nucleic Acids Res 33:4995-5005

16. Lapik YR, Fernandes CJ, Lau LF, Pestov DG (2004) Physical and functional interaction between Pes1 and Bop1 in mammalian ribosome biogenesis. Mol Cell 15:17-29

17. Hadjiolova KV, Nicoloso M, Mazan S, Hadjiolov AA, Bachellerie JP (1993) Alternative pre-rRNA processing pathways in human cells and their alteration by cycloheximide inhibition of protein synthesis. Eur J Biochem 212:211-215

18. Milkereit P, Strauss D, Bassler J, Gadal O, Kühn H, Schütz S, Gas N, Lechner J, Hurt E, Tschochner H (2003) A Noc complex specifically involved in the formation and nuclear export of ribosomal 40S subunits. J Biol Chem 278:4072-4081

19. Baßler J, Grandi P, Gadal O, Leßmann T, Petfalski E, Tollervey D, Lechner J, Hurt E (2001) Identification of a 60S preribosomal particle that is closely linked to nuclear export. Mol Cell 8:517-529

20. Henras AK, Soudet J, Gérus M, Lebaron S, Caizergue-Ferrer M, Mougin A, Henry Y (2008) The post-transcriptional steps of eukaryotic ribosome biogenesis. Cell Mol Life Sci 65:2334-2359 
21. Senger B, Lafontaine DL, Graindorge JS, Gadal O, Camasses A, Sanni A, Garnier JM, Breitenbach M, Hurt E, Fasiolo F (2001) The nucle(ol)ar Tif6p and Efl1p are required for a late cytoplasmic step of ribosome synthesis. Mol Cell 8:1363-1373

22. Barbieri R, Izzo V, Cantone M, Duro G, Giudice G (1992) Regulation of ribosomal RNA synthesis in sea urchin embryos. Rend Fis Acc Lincei 3:369-374

23. Cantone M, Barbieri R, Duro G, Izzo V, Giudice G (1993) Sequence analysis of the rDNA spacer of Paracentrotus lividus and observations about pre-rRNA processing. Mol Biol Rep 18:177-182

24. Davidson EH (1986) In gene activity in early development, 3rd edn. Academic Press, New York

25. Angerer LM, Yang Q, Liesveld J, Kingsley PD, Angerer RC (1992) Tissue-restricted accumulation of a ribosomal protein mRNA is not coordinated with rRNA transcription and precedes growth of the sea urchin pluteus larva. Dev Biol 149:27-40

26. Chomczynski P, Sacchi N (1987) Single-step method of RNA isolation by acid guanidinium thiocyanate-phenol-chloroform extraction. Anal Biochem 162:6-159

27. Sambrook J, Fritsch EF, Maniatis T (1989) Molecular cloning: a laboratory manual, 2nd edn. Cold Spring Harbor Press, Cold Spring Harbour, New York, USA

28. Asaro MR, Izzo V, Barbieri R (1999) Modified apparatus for voltage gradient gel electrophoresis. J Chromatogr A 855:723-726

29. Barbieri R, Izzo V, Costa MA, Giudice G, Duro G (1993) Voltage gradient electrophoresis of nucleic acids on agarose gels. Anal Biochem 212:168-172

30. Duro G, Barbieri R, Ribaudo MR, Feo S, Izzo V (1995) A downward capillary blotting procedure from hundred base pairs to hundred kilobases nucleic acids. Anal Biochem 225:360-362

31. Izzo V, Costa MA, Di Fiore R, Duro G, Bellavia D, Cascone E, Colombo P, Gioviale MC, Barbieri R (2006) Electrophoresis of proteins and DNA on horizontal sodium dodecyl sulfate polyacrylamide gels. Immun Ageing 12:3-7
32. Minokawa T, Rast JP, Arenas-Mena C, Franco CB, Davidson EH (2004) Expression patterns of four different regulatory genes that function during sea urchin development. Gene Expr Patterns 4:449-456

33. Ransik A, Davidson EH (1995) Micromeres are required for normal vegetal plate specification in sea urchin embryos. Development 121:3215-3222

34. Nemer M, Infante AA (1967) Ribosomal ribonucleic acid of the sea urchin egg and its fate during embryogenesis. J Mol Biol 27:73-86

35. Tocco G, Orengo A, Scarano E (1963) Ribonucleic acids in the early embryonic development of the sea urchin. I. Quantitative variations and 32-P orthophosphate incorporation studies of the RNA of subcellular fractions. Exp Cell Res 31:52-60

36. Rouquette J, Choesmel V, Gleizes PE (2005) Nuclear export and cytoplasmic processing of precursors to the $40 \mathrm{~S}$ ribosomal subunits in mammalian cells. EMBO J 24:2862-2872

37. Fatica A, Oeffinger M, Tollervey D, Bozzoni I (2003) Cic1p/ $\mathrm{Nsa3p}$ is required for synthesis and nuclear export of $60 \mathrm{~S}$ ribosomal subunits. RNA 9:1431-1436

38. Gadal O, Strauß D, Kessl J, Trumpower B, Tollervey D, Hurt E (2001) Nuclear export of $60 \mathrm{~S}$ ribosomal subunits depends on Xpolp and requires a nuclear export sequence-containing factor, $\mathrm{Nmd} 3 \mathrm{p}$, that associates with the large subunit protein Rpl10p. Mol Cell Biol 21:3405-3415

39. Ho J, Johnson AW (1999) NMD3 encodes an essential cytoplasmic protein required for stable 60S ribosomal subunits in Saccharomyces cerevisiae. Mol Cell Biol 19:2389-2399

40. Moy TI, Silver PA (1999) Nuclear export of the small ribosomal subunit requires the ran-GTPase cycle and certain nucleoporins. Genes Dev 13:2118-2133

41. Moy TI, Silver PA (2002) Requirements for the nuclear export of the small ribosomal subunit. J Cell Sci 115:2985-2995 